\title{
Nach Erstereignis einer TVT besteht langjähriges Risiko für PTS
}

Ende-Verhaar YM et al. Post-thrombotic syndrome: Short and long-term incidence and risk factors. Thromb Res 2019; 177: 102-109

Trotz antikoagulativer Therapie entwickeln ungefähr 20-50\% der Patienten mit einer tiefen Beinvenenthrombose (TVT) ein postthrombotisches Syndrom (PTS). Das Spektrum der Symptome beim PTS ist vielfältig, die langfristige Entwicklung und Inzidenz sind unbekannt. Die Untersuchung der langfristigen Entwicklung der Symptomatik des PTS ist Gegenstand der Studie.

Für ihre Analyse nutzte die niederländische Arbeitsgruppe Daten von Patienten der MEGA-Studie mit dem Erstereignis einer TVT. Die zwischen 1999 und 2004 durchgeführte MEGA-Studie war eine populationsbasierte Fall-Kontroll-Studie mit 4956 Patienten zwischen 18 und 70 Jahren mit Erstereignis einer venösen Thrombose (VT). Die meisten Betroffenen erhielten über einen Zeitraum von 3-6 Monaten eine antikoagulative Therapie. Nach 1 Jahr erfolgte eine Nachuntersuchung. Sowohl nach einem Jahr als auch nach 8 Jahren wurden jeweils in einem Fragebogen die Risikofaktoren für ein VT-Rezidiv sowie Symptome für ein PTS erfragt. Zwischen den Basischarakteristiken der Patienten, die die Fragebögen nach einem Jahr und nach 8 Jahren vollständig beantworteten, gab es keine Unterschiede. Die Diagnose eines PTS erfolgte auf der Basis des Villalta-Scores.
Bei 3153 Patienten der MEGA-Studie wurde die Diagnose einer TVT gestellt. Bei 1657 von ihnen (52,6\%) lag nach einem Jahr ein komplett beantworteter Fragebogen bezüglich der PTS-Symptomatik vor. Auf dieser Grundlage wurde bei 361 Betroffenen ein PTS diagnostiziert. Die kumulative Inzidenz über 1 Jahr betrug somit 2,18\% (95\%-KI 19,9-23,8), bei 92 Personen $(5,6 \%)$ bestand ein schweres PTS, bei $47(2,8 \%)$ ein Ulkus. Die am häufigsten berichteten Symptome waren schwere Beine (37\%), Schwellungen des Fußes oder der Wade (35\%) und Spontanschmerz in der Wade (26\%).

Nach 8 Jahren war von 633 Patienten ohne vorher bekanntes PTS ein komplett beantworteter entsprechender Fragebogen verfügbar. Bei 44 von ihnen wurde ein PTS diagnostiziert mit einer kumulativen Inzidenz zwischen einem und 8 Jahren von $7 \%$ (95\%-KI 5,2-9,2). Bei 13/44 (2,1\%) bestand ein schweres PTS, bei $12(1,9 \%)$ ein Ulkus. Die häufigsten Symptome waren neu entstandene Varizen (30\%), schwere Beine (22\%) und Schwellung von Fuß oder Wade (21\%).

Während des Follow-ups besserte sich die Symptomatik bei $69 \%$ und verschlechterte sich bei $7 \%$ der Patienten. Als Risikofaktoren nach einem Jahr erwiesen sich weibliches Geschlecht (RR 1,54; 95 \%-KI 1,2-
1,9) und Fettleibigkeit (RR 1,5; $95 \%-\mathrm{KI}$ 1,2-7,9), desgleichen im selben Ausmaß nach 8 Jahren. Weder nach einem Jahr noch nach 8 Jahren wurde das Risiko für ein PTS durch die Faktoren provozierte/unprovozierte TVT, Lokalisation des Thrombus, Schwangerschaft, Hormontherapie oder verschiedene Laborparameter beeinflusst.

\section{FAZIT}

Ihre Studie zeige, so die Autoren, dass bis zu 8 Jahre nach einer ersten TVT ein substanzielles Risiko für ein PTS besteht, am höchsten bei weiblichem Geschlecht und bei Fettleibigkeit. Bei fast $70 \%$ der Patienten besserte sich die PTS-Symptomatik. Einschränkend sei zu berücksichtigen, dass die Diagnose eines PTS nach dem VillaltaScore auf der Basis von Patientenfragebögen gestellt wurde und nicht nach klinischer Untersuchung und dass aufgrund der relativ niedrigen Rückläuferquote der Fragebögen ein signifikanter Selektionsbias aufgetreten sein könnte.

Dr. Gabriele Dobler, Berlin 